Article

\title{
Overexpression of Kif1A in the Developing Drosophila Heart Causes Valvar and Contractility Defects: Implications for Human Congenital Heart Disease
}

\author{
Takeshi Akasaka ${ }^{1,2,+}$, Karen Ocorr ${ }^{1, *}{ }^{\dagger}$, Lizhu Lin ${ }^{3}$, Georg Vogler ${ }^{1}$, Rolf Bodmer ${ }^{1}$ \\ and Paul Grossfeld ${ }^{3, *}$ \\ 1 Development, Aging \& Regeneration Program, Sanford Burnham Prebys Medical Discovery Institute, \\ La Jolla, CA 92037, USA; akasaka-cba@umin.ac.jp (T.A.); gvogler@sbpdiscovery.org (G.V.); \\ rolf@sbpdiscovery.org (R.B.) \\ 2 Cardiology and Catheterization Laboratories, Shonan Fujisawa Tokushukai Hospital, \\ Fujisawa City 251-0041, Japan \\ 3 Division of Cardiology, Department of Pediatrics, UCSD School of Medicine, San Diego, CA 92093, USA; \\ lilin@health.ucsd.edu \\ * Correspondence: kocorr@sbpdiscovery.org (K.O.); pgrossfeld@ucsd.edu (P.G.); \\ Tel.: +1-858-795-5125 (K.O.); +1-858-966-5855 (P.G.) \\ + These authors contributed equally to this manuscript.
}

Academic Editor: Andy Wessels

Received: 13 May 2020; Accepted: 26 May 2020; Published: 2 June 2020

\begin{abstract}
Left-sided congenital heart defects (CHDs) are among the most common forms of congenital heart disease, but a disease-causing gene has only been identified in a minority of cases. Here, we identified a candidate gene for CHDs, KIF1A, that was associated with a chromosomal balanced translocation $\mathrm{t}(2 ; 8)(\mathrm{q} 37 ; \mathrm{p} 11)$ in a patient with left-sided heart and aortic valve defects. The breakpoint was in the $5^{\prime}$ untranslated region of the KIF1A gene at 2q37, which suggested that the break affected the levels of Kif1 $\mathrm{A}$ gene expression. Transgenic fly lines overexpressing Kif1 $\mathrm{A}$ specifically in the heart muscle (or all muscles) caused diminished cardiac contractility, myofibrillar disorganization, and heart valve defects, whereas cardiac knockdown had no effect on heart structure or function. Overexpression of Kif1 $A$ also caused increased collagen IV deposition in the fibrous network that normally surrounds the fly heart. Kif $1 A$ overexpression in $\mathrm{C} 2 \mathrm{C} 12$ myoblasts resulted in specific displacement of the F-actin fibers, probably through a direct interaction with G-actin. These results point to a Kif1A-mediated disruption of F-actin organization as a potential mechanism for the pathogenesis in at least some human CHDs.
\end{abstract}

Keywords: Kif1A; cardiac development; congenital heart disease; aortic valve; Drosophila; myoblasts

\section{Introduction}

Congenital heart defects are the most common birth defect, occurring in about $0.7 \%$ of all newborn infants [1]. Left-sided heart defects comprise one of the most common forms of congenital heart disease and have a strong genetic component [2]. One of the most severe heart defects, hypoplastic left heart syndrome (HLHS), occurs in $2-4 \%$ of all infants born with congenital heart disease, and there are 1000-2000 infants diagnosed each in year in the United States with HLHS [3]. Despite significant strides in palliative surgical approaches for HLHS, in which the right ventricle is converted to function as the systemic ventricle, HLHS still accounts for $20-25 \%$ of mortality in all infants born with congenital heart disease. The long-term prognosis for survivors remains guarded, and it is likely that many patients will ultimately require cardiac transplantation. 
Although there are multiple lines of evidence supporting a genetic etiology for left-sided heart defects, the underlying molecular and cellular mechanisms are poorly understood. The most commonly held theory is that, in HLHS, left ventricular hypoplasia results secondarily due to hemodynamic perturbations, specifically decreased anterograde blood flow into the developing left ventricle, secondary to obstruction of the mitral and/or aortic valves. In support of this hypothesis, ligation of the left atrium in the chick embryo decreased left ventricular inflow and causes hypoplasia of the left ventricle [4]. Alternatively, in some cases, HLHS may be due to a primary defect in left ventricular development. In this case, HLHS may represent a severe neonatal form of cardiomyopathy, suggesting an underlying defect in cardiac myocyte development. Consistent with this model, histopathologic analysis of left ventricular myocardial tissue from explanted HLHS infant hearts revealed fibrosis and myocyte disarray [5], as observed in some forms of cardiomyopathy, although it is possible that these changes arise secondarily to impaired hemodynamics. Interestingly, mutations in the cardiac transcription factor NKX2.5 have been observed in patients with HLHS [6] and in other patients with hypertrophic cardiomyopathy (HCM) [7], suggesting that HLHS and HCM could represent different forms of cardiomyopathy along a common disease spectrum. In this case, it is possible that multiple developmental pathways are affected that cause primary valve and myocardial defects through a "multi-hit" mechanism (either through a single or multiple affected genes) and that additional genetic and/or epigenetic factors can influence the severity of the phenotype.

In this study, we identified a patient with left-sided congenital heart defects who carried a balanced chromosomal translocation $\mathrm{t}(2 ; 8)$ (q37;p11). We localized the chromosome 2 breakpoint to the $5^{\prime}$ putative regulatory region of the KIF1A gene. The KIF protein superfamily comprises microtubule-associated molecular motors that are critical for intracellular transport [8-10]. Mutations in other KIF family members (KIF3A and KIF7) have been associated with sinus inversus and heterotaxy [11], suggesting that KIF proteins are essential for normal heart development and that impaired expression of other members of the KIF family might also cause congenital heart defects. In adults, Kif1 $A$ has been shown to be dysregulated following myocardial infarction [12] and a Kif1 $A$ motor domain mutation has been associated with spastic paraplegia [13]. We hypothesized that dysregulation of KIF1A expression contributed to the left-sided heart defects in our patient. In this study, we demonstrate that overexpression (OE) of Kif1 $A$ causes cardiac morphological and functional defects in the fly heart that are consistent with defects observed in F-actin organization and maturation of cultured mammalian myoblasts. Kif1 $A$ OE also altered bristle arrangement and ommatidium viability in the fly, suggesting an effect on cell polarity. Taken together, these data demonstrate that dysregulation of Kif1A expression may impair normal heart development via disruption of F-actin organization, suggesting a potentially novel mechanism for some of the most common forms of human congenital heart disease.

\section{Materials and Methods}

\subsection{Patient Consent}

Consent to obtain blood and to perform genetic studies was obtained through a University of California - San Diego Institutional Review Board-approved protocol.

\subsection{Analysis of Human Aortic Valve Tissue}

For histological analyses, human aortic valve was dissected out into single leaflet and fixed in $37 \%$ formalin, washed with Phosphate Buffered Saline (PBS), dehydrated with ethanol, embedded in paraffin, and cut into $10-\mu \mathrm{m}$-thick sections using a microtome. Sections were stained with hematoxylin-eosin, Masson's trichrome stain, and von korssa stain according to standard protocols. An age- and sex-matched control sample, obtained through the San Diego County Coroner's Office, was used from a patient who died from noncardiac causes. 


\subsection{Fly Stocks}

We used "driver" fly lines expressing the following alleles: twist-Gal4;24B-Gal4, GMH5, GMR-Gal4, pannier-Gal4, Mef2-Gal4, and Hand-Gal4. Overexpression of the mouse Kif1 A transgene was performed using the UAS-Gal4 system, where "driver"-Gal4 lines were crossed to transgenic lines containing an upstream activating (UAS) sequence and either mouse Kif1 $A$ or interfering RNA against unc-104 (UAS unc104 RNAi). The yw laboratory strain was used as a reference in this study. To generate the Kif1A transgenic flies, the mouse Kif1A was subcloned into the pUAST Drosophila transformant vector (details below) and then injected according to standard procedures. Three individual transformant lines were examined in this study (line 3, line 6, and line 8b). Unc-104 RNAi line (transformant ID \#23455) was obtained from the Vienna Drosophila RNAi center (Vienna, Austria).

\subsection{Fly Heart Staining and Image-Based Heart Performance Assay}

Flies were anaesthetized with FlyNap@ (Carolina Biological Supply Co, Burlington, NC, USA) followed by dissection in artificial hemolymph as previously described [14], and cardiac function was assayed using high speed digital imaging (100-150 fps, 9300 EM-CCD camera, Hamamatsu Corp., Bridgewater, NJ, USA) followed by movie analysis with the Semiautomatic Optical Heartbeat Analysis (SOHA; www.sohasoftware.com) method [15,16].

\subsection{Quantitative RT-PCR}

Total RNA was extracted from whole flies $(\mathrm{N}=6)$, or isolated thoraxes $(\mathrm{N}=10)$, abdomens $(\mathrm{N}=10)$, heads $(\mathrm{N}=20)$, intestines $(\mathrm{N}=20)$, and hearts $(\mathrm{N}=30)$ (the same number of male and female flies per group) using TRIzol (Invitrogen, Carlsbad, CA, USA) and purified with RNeasy kit (QIAGEN, Germantown, MD, USA) for adult fly or Mini RNA isolation kit (Zymo Research, Irvine, CA, USA) for heart RNA. After treatment with DNase I, first strand cDNA was transcribed by SuperScript III (Invitrogen, Carlsbad, CA, USA) by oligo(dT) primer, followed by second strand synthesis. Quantitative PCR was carried out using LightCycler FastStart DNA Master PLUS SYBR Green I kit (Sigma-Aldrich Corp., St. Louis, MO, USA). The following primer sets were used:

rp49: 5' - GACGCTTCAAGGGACAGTATCTG -3' 5' - AAACGCGGTTCTGCATGAG -3'

unc-104: 5' - GCGCTGCTATAACTCAG-3' 5' - GTTTGTTCCGTAACGTGTTG -3'

\subsection{Cloning of Full-Length and Truncated Mouse Kif1A}

The mouse Kif1A cDNA (mKif1A/pYX-Asc) was purchased from Clontech (Mountain View, CA, USA). Site-directed mutagenesis to convert the terminal codon into a BamHI site was performed by using the QuikChange Site-Directed Mutagenesis kit (Stratagene, La Jolla, CA, USA) using the following oligonucleotides (the base pair substitution is underlined):

5'-CTGCGCAGATGCGGGTCGGATCCGAAAGCCTTCCGGACTTC-3' and

\section{5'- GAAGTCCGGAAGGCTTTCGGATCCGACCCGCATCTGCGCAG -3'.}

Full-length Kif1A was subcloned into the EcoRI-BamH1 site of the pcDNA3.1 myc-His(-) vector to add the myc tag and then subcloned into the XbaI-Not1 site of the pUAST vector. Three truncated proteins containing the motor domain (MD), the forkhead associated domain (FAD), and the Pleckstrin homology domain $\mathrm{PH}$ were obtained using full-length mouse Kif1 $A$ as a template. The fragments were amplified by PCR using the following primer sets:

MD: 5'-CGAGAAATGGCCGGGGCCTCTG-3' and 5'GTCGACCAGCCAGCAAGGCTTCCCTCT-3' FAD: 5' - CTCGAGAGATGGGTGTGGCCATGAG-3' and 


\section{PH: 5' - CTCGAGGCATGGGGACTTTCCTTCTC-3' and}

\section{5'-GTCGACTCTGCGCAGATCTCCTTCGA-3'}

The PCR fragments were ligated into a pCR2.1-TOPO cloning vector (Invitrogen, Carlsbad, CA, USA) and sequenced to ensure no errors were introduced during the PCR. All the fragments of full-length and truncated Kif1A were then subcloned into the XhoI-NotI site of pcDNA3.1/myc-His(-)A expression vector (Invitrogen, Waltham, MA, USA) for cell transfection experiments.

\subsection{Cell Culture and Transfection}

C2C12 cells were cultured in Dulbecco's modified Eagle's medium (D-MEM) supplemented with $15 \%(v / v)$ heat-inactivated fetal bovine serum in a humidified $5 \% \mathrm{CO} 2$ incubator at $37{ }^{\circ} \mathrm{C}$. For transient transfections, $\mathrm{C} 2 \mathrm{C} 12$ cells were grown on collagen I coated cover slips in 6-well plate and transfected with $1 \mu \mathrm{g}$ of myc-tagged KifA expression plasmids using $4 \mu \mathrm{L}$ of FuGene 6 transfection reagent (Roche) according to the standard protocol provided by the manufacture. Thirty-six hours after transfection, cells were immunostained (see below). C2C12 cells were differentiated into myocytes at $24 \mathrm{~h}$ after transfection in D-MEM supplied with $2 \%(v / v)$ heat-inactivated horse serum and Insulin-Transferrin-Selenium liquid media supplement (Sigma). After $36 \mathrm{~h}$ in differentiation medium, the cells were immunostained (see below).

\subsection{Immunostaining}

Cells were fixed with $4 \%$ Paraformaldehyde for 15 min and permeabilized with $0.5 \%$ Triton X100 in PBS for 5 min at room temperature. Cells were blocked with 3\% Bovine Serum Albumin in PBS followed by primary antibody incubation at room temperature for $2 \mathrm{~h}$. Mouse anti-myc at 1:100 (Santa Cruz Biotechnology, Dallas, TX, USA), mouse anti-tublin at 1:500 (Sigma-Aldrich, St. Louis, MO, USA), and Mouse anti-GFP (Green Fluorescent Protein) at 1:250 (Invitrogen, Carlsbad, CA, USA) were used for primary antibodies. Cells were then incubated with FITC (Fluorescein isothiocyanate) -conjugated donkey anti-mouse IgG (1:200; Jackson ImmunoResearch Laboratory, West Grove, PA, USA) with Alexa Fluor 594-conjugated phalloidin (1:100; Invitrogen, Carlsbad, CA, USA) was at room temperature for $2 \mathrm{~h}$. Cells were mounted on glass slides with Vectashield (with DAPI), and the images were taken using a Fluoview 1000 Olympus Laser Point Scanning Confocal Microscope. Myocyte cell sizes and nuclei number were quantified from confocal images using Image J Trainable Weka Segmentation plugin.

Semi-intact fly heart preparations were prepared for immunohistochemistry by first incubating in $10 \mathrm{mM}$ EGTA (ethylene glycol-bis( $\beta$-aminoethyl ether)- $\mathrm{N}, \mathrm{N}, \mathrm{N}^{\prime}, \mathrm{N}^{\prime}$-tetraacetic acid) in artificial hemolymph for $5 \mathrm{~min}$ to arrest the heart in the diastolic phase. Then, hearts were fixed with $4 \%$ paraformaldehyde and processed as previously described [17]. Images were taken using a Mouse anti-pericardin (collagen IV) antibody (Developmental Studies Hybridoma Bank, Univ. of Iowa) at 1:100 for primary antibody, and cells were then incubated with 488 Alexa Fluor-conjugated goat anti-mouse IgG (1:200; Jackson ImmunoResearch Laboratory, West Grove, PA, USA) and with Alexa Fluor 594-conjugated phalloidin (1:100; Invitrogen, Carlsbad, CA, USA) to stain F-actin. Images were taken with a Zeiss ApoTome microscope (Carl Zeiss, White Plains, NY, USA). The pericardin area was quantified from $Z$ stack projections using Image J and the Trainable Weka Segmentation plugin.

\subsection{Immunoprecipitation and Western Blot}

HEK293T cells were co-transfected with $3 \mu \mathrm{g}$ of full-length or truncated myc-tagged Kif1 A and $3 \mu \mathrm{g}$ of GFP-tagged actin or GFP expression plasmids in 10-cm culture dish using $24 \mathrm{ugl}$ of FuGene6 (Sigma-Aldrich Corp., St. Louis, MO, USA).

Thirty-six hours after transfection, cells were lysed on ice for $30 \mathrm{~min}$ with a lysis buffer ( $20 \mathrm{mM}$ Tris PH7.6, $150 \mathrm{mM} \mathrm{NaCl}, 1 \% \mathrm{NP40}$, and $1 \mathrm{mM}$ EDTA, Ethylenediaminetetraacetic acid) supplemented with CompleteMini proteinase inhibitor (Sigma-Aldrich Corp., St. Louis, MO, USA). Lysates were centrifuged to remove the insoluble fraction and supernatants were pre-cleaned with Protein $G$ 
sepharose (Sigma-Aldrich Corp., St. Louis, MO, USA). The supernatants were then incubated with $1 \mu \mathrm{g}$ of rabbit anti-myc antibody (Santa Cruz Biotechnology Inc, Dallas, TX, USA) for $2 \mathrm{~h}$ at $4{ }^{\circ} \mathrm{C}$ followed by incubation with Protein G sepharose for one hour at $4{ }^{\circ} \mathrm{C}$. After 6 washes with lysis buffer, bound proteins were eluted at $70{ }^{\circ} \mathrm{C}$ for $5 \mathrm{~min}$ in NuPage LDS buffer (Invitrogen, Carlsbad, CA, USA). All samples were resolved on a 4-12\% SDS-PAGE gel and transferred to the Hybond ECL nitrocellulose filter (Sigma-Aldrich Corp., St. Louis, MO, USA). After blocking with Tris Buffered Saline with Tween (50 mM Tris-Cl PH7.6, $150 \mathrm{mM} \mathrm{NaCl}$, and 0.05\% Tween20) containing 3\% BSA, the blots were incubated with mouse ant-GFP antibody (Takara Bio, Mountain View, CA, USA) at 1:4000 or mouse anti-myc (Invitrogen, Carlsbad, CA, USA) at 1:4000 overnight at $4{ }^{\circ} \mathrm{C}$, followed by the horse raddish peroxidase (HRP)-conjugated anti-mouse IgG (Sigma-Aldrich Corp., St. Louis, MO, USA) at 1:4000 for $1.5 \mathrm{~h}$ at room temperature. The specific proteins were detected using ECL plus (Sigma-Aldrich Corp. St. Louis, MO, USA).

\section{Results}

\subsection{Molecular Mapping of the $t(2,8 ; q 37, p 11)$ Chromosomal Translocation Breakpoint in a Patient with} Left-Sided Congenital Heart Defects

We identified a patient with left-sided heart defects in association with a balanced chromosome translocation $(\mathrm{t}(2,8 ; \mathrm{q} 37, \mathrm{p} 11)$. He was born with coarctation of the aorta and aortic valve stenosis. He underwent surgical repair for coarctation at age seven years in 1968, aortic valve replacement with a human homograft at age 29 years in 1990, and then again at age 49 years in 2009. He carries a balanced chromosomal translocation ( $\mathrm{t}(2,8), 2 \mathrm{q} 37 ; 8 \mathrm{p} 11)$ (Figure 1A). Previous reports have associated left-sided heart defects with chromosomal deletions spanning 2q37, and consequently, we hypothesized that this patient's 2q37 translocation breakpoint might affect the expression of a causal gene for left-sided heart defects. Therefore, in this study, we did not assess candidate genes at the $8 \mathrm{p} 11$ breakpoint. Toward that end, we performed molecular mapping of the $2 \mathrm{q} 37$ translocation breakpoint by fluorescence in situ hybridization on the patient, which localized the chromosome $2 q 37$ breakpoint to a 14-kb region $18 \mathrm{~kb}$ upstream of the transcription initiation site of the Kif1 $A$ gene [18]. Furthermore, although there are other genes in the region of the 2q37 breakpoint, we focused on the Kif $1 A$ gene, given its proximity to the breakpoint. We obtained paraffin-embedded tissue from the patient's explanted aortic valve from his second surgery in 1990 for analysis. The leaflets of the patient's aortic valve were noticeably thickened (Figure 1B) compared to valve leaflet tissue from an age- and sex-matched normal control (Figure 1C). Histological analysis of the patient's aortic valve leaflets revealed a dramatic loss of interstitial cells (Figure 1D compared to control in Figure 1E), replaced by diffuse fibrosis (Figure 1D' compared to control in Figure 1E') and severe calcification compared to the control (Figure 1D", $\mathrm{E}^{\prime \prime}$, respectively).

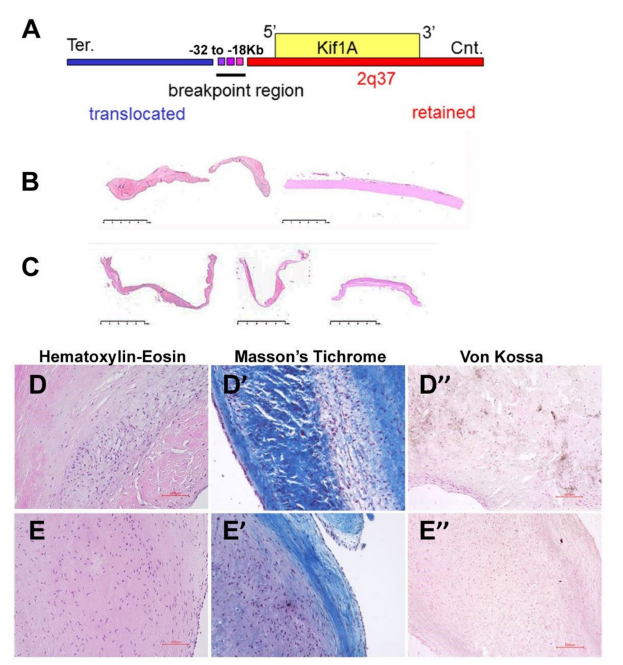

Figure 1. Patient with left-sided heart defects associated with a $t(2,8 ; q 37, p 11)$ balanced translocation: 
(A) Molecular mapping of the 2q37 translocation breakpoint by fluorescence in situ hybridization. The transcription initiation site of the KIF1A gene is downstream of the breakpoint region. Whole mount, transverse sections of explanted aortic valve leaflets from $(\mathbf{B})$ the patient and $(\mathbf{C})$ an age- and sex-matched unaffected control. (D,E) Higher magnification images of sections from explanted aortic valve tissue from the patient $\left(\mathbf{D}, \mathbf{D}^{\prime \prime}\right)$ and an age and sex-matched unaffected control $\left(\mathbf{E}, \mathbf{E}^{\prime \prime}\right)$. D and E: Hematoxylin-Eosin stain showing interstitial cells; D' and E': Masson's Trichrome stain showing loss of interstitial cells accompanied by increased fibrosis (blue) in the patient; and $\mathbf{D}^{\prime \prime}$ and $\mathbf{E}^{\prime \prime}$ : Von Kossa stain showing calcification (brown) in the patient.

\subsection{Kif1A Overexpression Impairs Cardiac Function}

Recent studies have demonstrated that manipulations of human cardiac disease gene homologs in Drosophila can reproduce human cardiac phenotypes in the fly heart [14,19-22]. We reasoned that a breakpoint close to the KIF1A transcription start site might affect gene expression either by increasing or decreasing transcription. Kif1Ais normally expressed in neuronal tissues in the mouse but has not been shown to be expressed in the heart [23]. We initially examined the effect of loss of Kif1A function on heart function in the adult fly using the Gal4-UAS system [24]. We used RNAi technology $[17,24]$ to knockdown (KD) unc-104, the functional counterpart of Kif1A that exists as a single gene in the Drosophila genome. We examined heart function in mesoderm-specific unc-104 KD flies and in heterozygous deficiency flies (Df(2R)Exel6064/w1118, Df(2R)Exel7145/w1118) using high-speed video imaging of hearts in a semi-intact preparation [15]. We found that heart function was unaffected in either cardiac-specific KD or deficiency flies and was similar to that observed in laboratory wildtype flies with the matched genetic backgrounds (Supplementary Table S1), suggesting that unc-104 is not essential for normal heart development and adult function in the Drosophila system. This is also supported by the fact that normally unc-104 expression in the heart is almost undetectable (Supplementary Figure S1) and that no cardiac phenotype has been observed in Kif1 A knockout mice [25].

We next tested the possibility that the breakpoint results in overexpression of Kif1A to affect heart function. We generated transgenic fly lines that overexpress the mouse Kif1A protein tagged with myc. Using the Gal4-UAS system, we drove overexpression specifically in mesoderm (using twist;24B-Gal4, 24B-Gal4, and mef2-Gal4) or specifically in myocardial cells (using GMH5-Gal4). Exogenous expression of mouse Kif1 $A$ was confirmed by Western blotting using anti-myc antibody (Supplementary Figure S2A). Hearts from 3-week-old Kif1A overexpressing flies demonstrated normal diastolic and systolic intervals (Supplementary Table S1), but in all cases, these hearts exhibited significant reductions in contractile function (measured as \% fractional shortening, Figure 2A, Supplementary Table S1). Similarly, overexpression of the fly Kif1A, unc104, also reduced cardiac contractility (Figure 2A, right bars). Interestingly, in 1-week-old flies, contractility was not affected when Kif1A was overexpressed using a relatively weak cardiac-specific driver GMH5-Gal4 (Supplementary Table S1). However, by three weeks of age, cardiac contractility in these flies was strikingly compromised (Figure 2A), suggesting that Kif1 $A$ overexpression causes dose-dependent and progressive cardiac dysfunction with age. A dose dependency is also suggested by the increasingly severe reduction in fractional shortening as the strength of the driver increases (Figure 2A). For the strongest drivers used (24B-Gal4 and twist;24B-Gal4), this decreased contractility was correlated with an apparent dose-dependent increase in end systolic diameters (Supplementary Figure S3A) and less consistent decreases in the end diastolic diameters (Supplementary Figure S3B), indicating systolic dysfunction. 


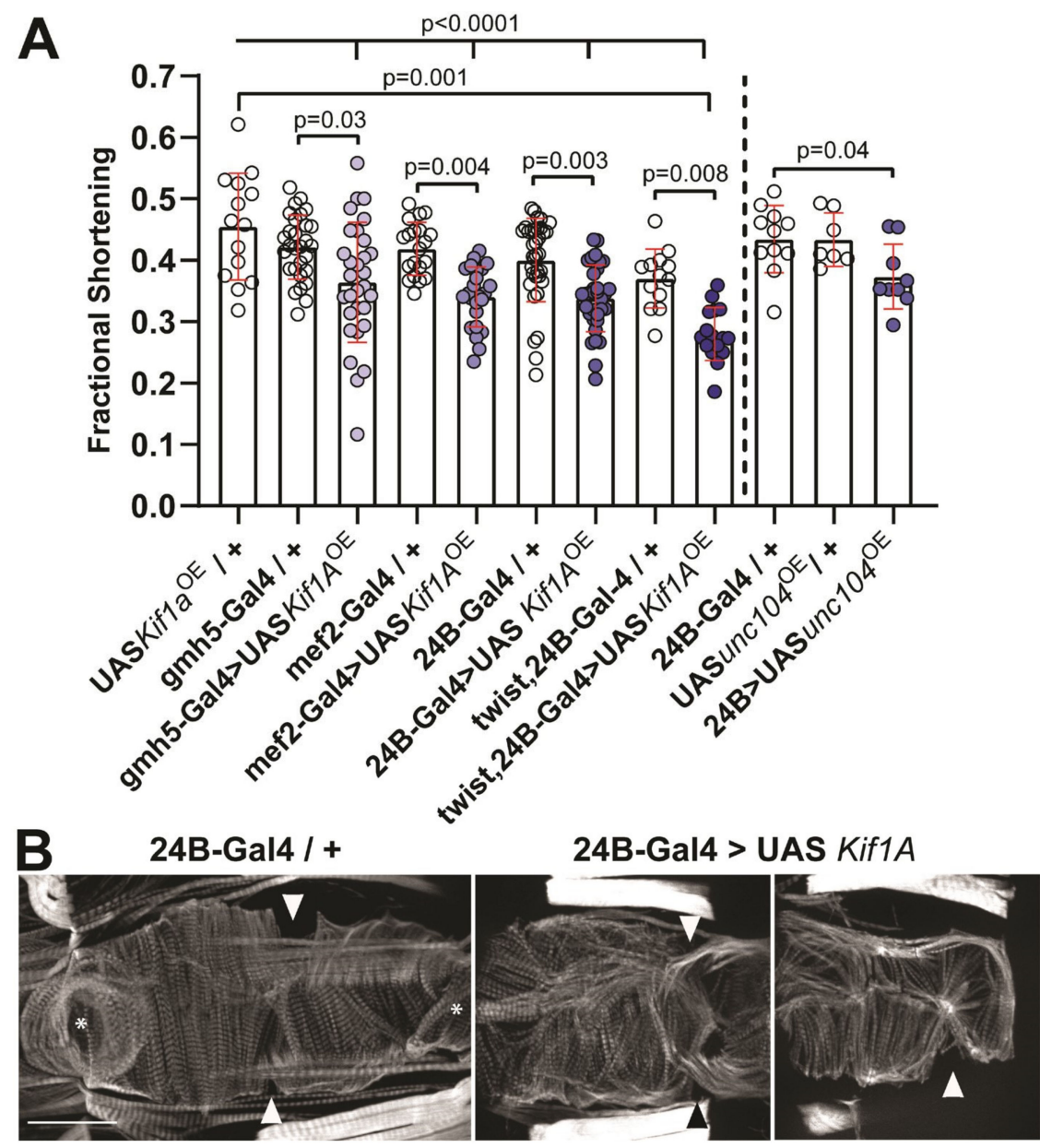

Figure 2. Kif1 $A$ overexpression causes reduced contractility and increased myofibrillar disarray: (A) Mesodermal (Twist;24B-Gal4 and 24B-Gal4, and mef2-Gal4) and cardiac-specific (GMH5-Gal4) drivers were used to drive (left) Kif1 A and (right) unc104 overexpression in the hearts of 3-week-old flies. In all cases, overexpression of mouse Kif1A or fly unc104 significantly reduced fractional shortening, a measure of cardiac contractility, compared to controls. Significance was determined using a one-way analysis of variance and Tukey's multiple comparisons post hoc test; number of flies in each group is shown in Supplementary Table S1. (B) Hearts from 3-week-old adult flies stained for F-actin: Control hearts (24B-Gal4/+) show compact myofibrillar structure and well-defined ostia (inflow tracts, arrowhead) and valve (asterisk). Kif1A overexpression resulted in hearts with primarily thin and disorganized myofibrils within the cardiac myocytes with many gaps due to myofibrillar disarray. For orientation purposes, the location of the inflow tracts is indicated by arrowheads. Scale bar is 50 microns; anterior is to the left.

\subsection{Kif1A Overexpression Impairs Cardiac Morphology}

We examined the morphology of adult hearts with Kif1A overexpression. Hearts in adult Drosophila are located just below the cuticle on the dorsal side of the abdomen and are composed of three distinguishable muscles: circumferential muscles, longitudinal muscles, and alary muscles [26,27]. The circumferential muscles are the working myocardial cells. Hearts were stained with fluorescent phalloidin to visualize the filamentous actin (F-actin) structure in the myofibrils. In controls, the myofibrils are circumferentially arranged to facilitate squeezing of the heart tube and hemolymph ejection (Figure 2B, 24B-Gal4/+). In hearts from all Kif1A overexpressing flies, myofibrils were not tightly packed, exhibited significant disarray within the myocardial cells (Figure 2B, 24B-Gal4>Kif1A, see also Figure 3), and were consistent with the observed reductions in contractility. 

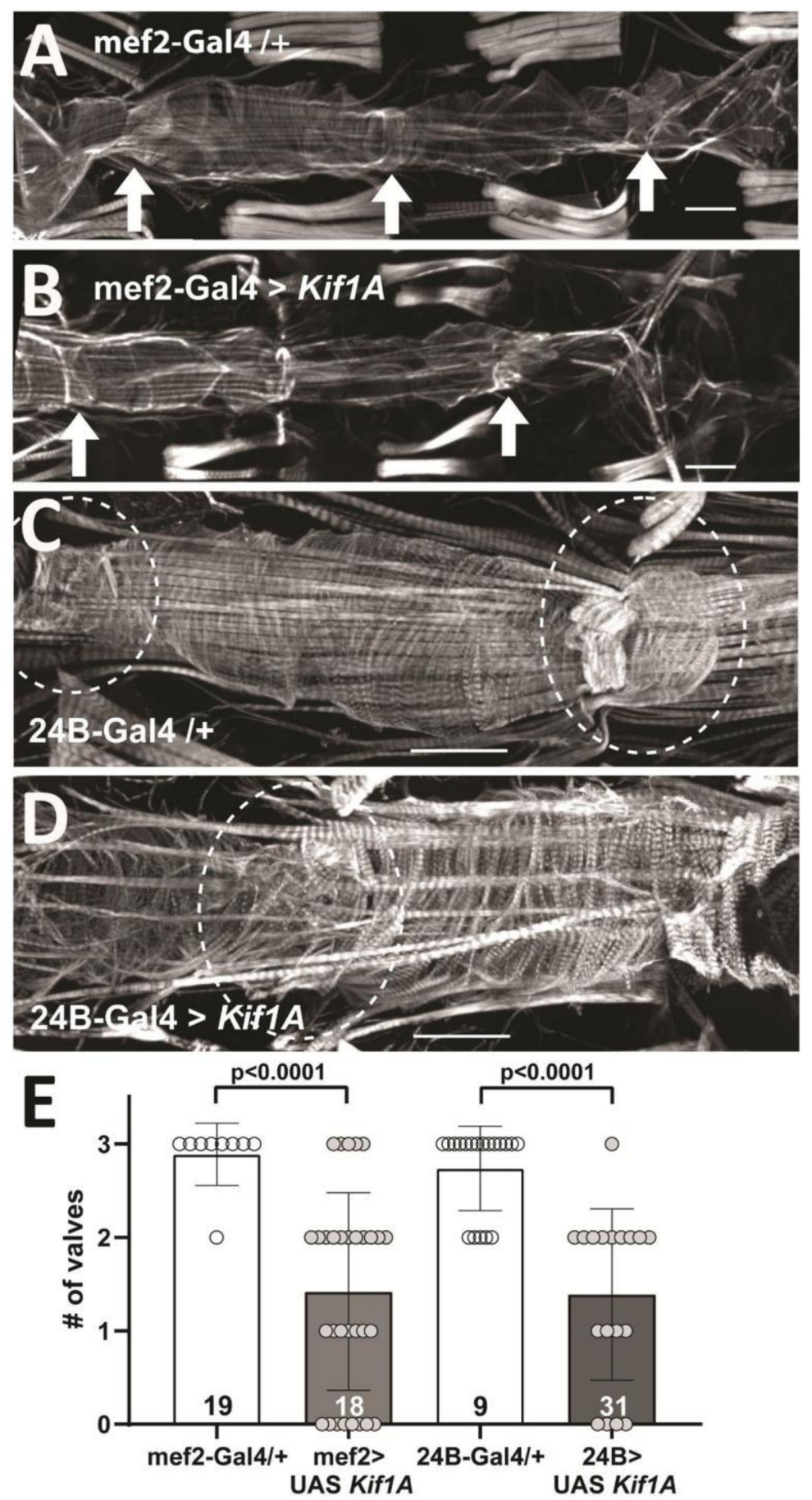

Figure 3. Kif1 $A$ overexpression causes valve remodeling: (A) F-actin staining with phalloidin of a control heart (mef2-Gal4/+) showing circumferential arrangement of myofibrils in the heart chambers and a denser myofibrillar arrangement in the 3 valve structures (indicated by arrows). Scale bar is 50 microns; anterior is to the left in all pictures. (B) In Kif1A overexpressing (OE) hearts (mef2-Gal4 > UAS Kif1A), the 2 identifiable valves (indicated by arrows) show reduced myofibrillar structure. (C) F-actin staining in a control heart (24B-Gal4/+) shows dense myofibrillar structure in the valve (dashed line circle). (D) Staining in Kif1A OE hearts (24B-Gal4 > UASKif1A OE) shows disorganized and loosely arranged myofibrils in the valve. Note overall reduction in F-actin containing myofibrils in Kif1A OE hearts in both Figure 3B,D. (E) Quantitation of identifiable valves in controls (24B-Gal4/+ and mef2-Gal4/+) and in F-actin stained hearts from Kif1A OE flies (24B > UAS KifAa and mef2 > UAS Kif1A): The number of hearts is indicated in each bar; ${ }^{* * * *} p<0.0001$, Mann-Whitney test. 
Using SDS-PAGE electrophoresis, we confirmed a significant reduction in myosin heavy chain protein in isolated hearts overexpressing Kif1A (Hand-Gal4 > UAS-Kif1A) compared to controls (Supplementary Figure S2B). These findings indicate that Kif1A overexpression disrupts the myofibrillar structure and reduces cardiac muscle protein content.

In addition, we noted that hearts overexpressing Kif1 $A$ also exhibited valve dysfunction compared to controls (Supplementary Video S1). The linear fly heart is normally divided into four chambers by the presence of three internal valves with valve cellular structure characterized by a dense arrangement of myofibrils (Figure 3A, arrows). However, in flies where Kif1 A is overexpressed in the heart, some valves appeared to be absent (Figure 3B, Supplementary Video S1, bottom two hearts). In addition, the arrangement of myofibrils in the valves was disorganized and diffuse (compare Figure 3C,D). Quantitation of identifiable valves from phalloidin stained preparations showed a significant reduction in the number of well-defined valves in Kif1A overexpressing flies compared to controls (Figure 3E).

We also examined the presence and arrangement of the extracellular collagen network that normally surrounds the fly heart. One of the primary components of this network is pericardin, a type IV collagen, which can be visualized by anti-pericardin staining revealing a "fishnet" arrangement of collagen fibers that surrounds and supports the heart tube. Hearts overexpressing Kif1 A exhibited an increase in the number and thickness of collagen fibers (Figure $4 \mathrm{~A}-\mathrm{C}$ ). Quantification of the area of collagen surrounding the heart showed a significant increase in hearts where Kif1 $A$ was overexpressed using the stronger 24B muscle driver, again suggesting a dose-dependent effect (Figure 4C).
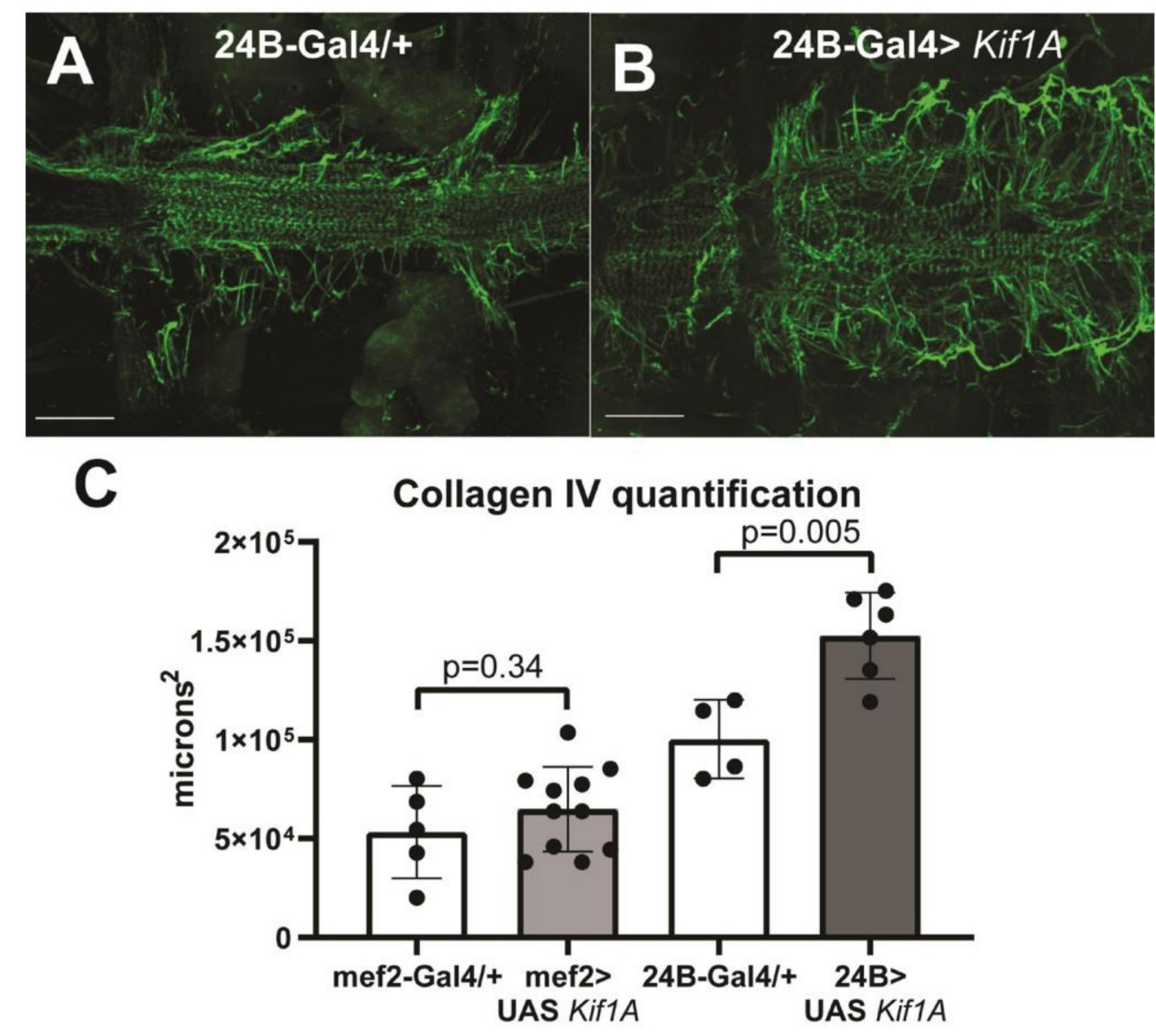

Figure 4. Kif1A overexpression increases collagen IV deposition: (A) Anti-pericardin (collagen IV homolog) staining reveals the extracellular matrix structure surrounding the cardiac tube in control flies expressing the muscle cell driver 24B-Gal4. (B) Muscle-specific Kif1A overexpression caused a significant increase in the extent of cardiac-associated collagen IV network. Scale bar is 50 microns; anterior is to the left. (C) Quantification of the ECM networks from Z-stacks shows significant increases in response to muscle cell-specific Kif1A OE. Significance was determined by two-tail, unpaired T-tests. 


\subsection{Kif1A Overexpression Does Not Affect Early Cardiac Development}

To test whether the effect of Kif1A overexpression on heart function/morphology occurred during Drosophila embryological heart development, we analyzed the cardiac precursor cell number and the distribution of the cell polarity marker Dlg (disk large), which is normally localized to the dorsal-lateral sides of the heart. Analysis of stage 18 embryos revealed no differences in overall cell number and in the Dlg localization between wild-type and Kif1 $A$ overexpressing stage 18 embryos (Supplementary Figure S4), indicating that overexpression of Kif1A does not affect cardiomyocyte commitment or cell polarity in early stages of Drosophila development.

\subsection{Overexpression of Kif1A Affects Actin Fiber Distribution and Mammalian Cell Morphology}

Based on our results in the fly model, we aimed to determine if overexpression of Kif1 $\mathrm{A}$ also affects morphology in mammalian muscle cells. Kif1 $A$ contains three known domains: the kinesin motor domain (MD), the forkhead associated domain (FHA), and the Pleckstrin homology domain (PH) (Figure 5F). In neurons, the MD interacts with and moves anterograde along microtubules using energy from microtubule-stimulated ATP hydrolysis. The FHA domain plays an important role in stabilizing the monomeric form of the Kif1A protein, which is critical for Kif1A motor activity [28]. The PH domain is known to physically associate with the lipid membrane of neurotransmitter vesicles [29].
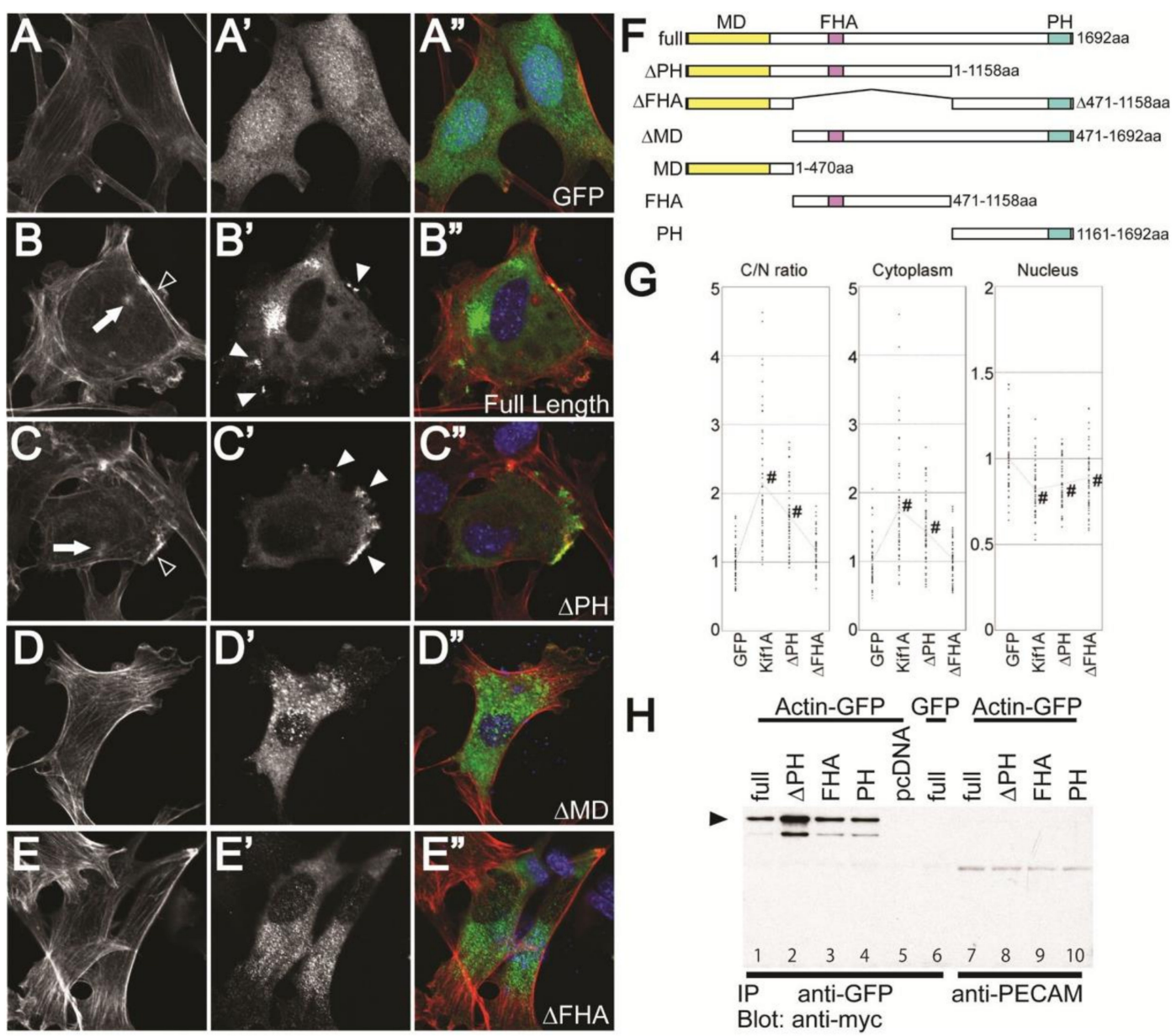

$\mathrm{H}$

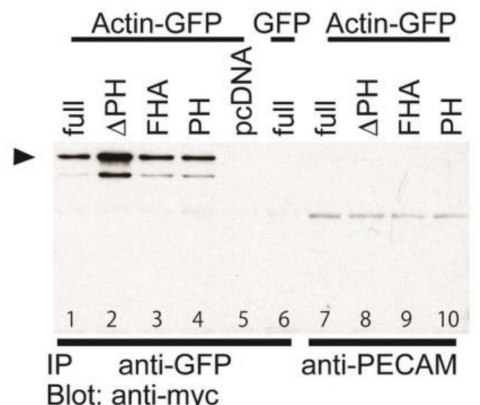

Figure 5. Actin cytoskeleton is affected in Kif1A-transfected C2C12 myoblasts: (A-E) Phalloidin staining reveals F-actin organization in transfected C2C12 cells. $\alpha-$ GFP or $\alpha$-myc staining identifies

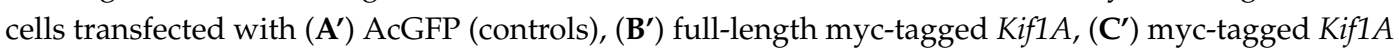

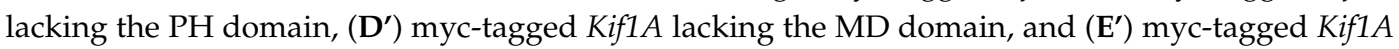
lacking the FHA domain. The F-actin organization is displaced more towards the periphery of the cell 
(open arrowheads) where there are aggregates of Kif1A (solid arrowheads), as shown in Figure 5B,C. This effect is observed in the construct lacking the lacking the PH domain but not in those lacking the motor domain or the forkhead-associated domain, implying that normal motor domain function is required for overexpressed Kif1A protein to exert its effect. (F) Schematic illustrating the structure of full-length and truncated Kif1A proteins. MD: kinesin motor domain, FHA: forkhead associated domain, and PH: Pleckstrin homology domain. All the constructs listed in Figure 5F were tagged with myc for detection; (G) Kif1a transfection increases cell size. Two-dimensional cell surface area was measured in $\mathrm{C} 2 \mathrm{C} 12$ cells transfected with AcGFP, full-length Kif1A, truncated Kif1A $\triangle \mathrm{FHA}$, or $\triangle \mathrm{PH}$. The area occupied by cytoplasm in Kif1A-transfected cells is significantly larger than control (Ac-GFP). Numbers of cells analyzed: GFP $=54, K i f 1 A=51, \Delta \mathrm{PH}=53$, and $\triangle \mathrm{FHA}=52$; non-repeated measures ANOVA followed by Student-Newman-Keuls post hoc test. \#p $<0.01$ compared to GFP-transfected cells. (H) Lysate from C2C12 cells co-transfected with actin-GFP and full-length (full) or truncated Kif1A $(\triangle \mathrm{PH}, \mathrm{FHA}$, and $\mathrm{PH})$ were immunoprecipitated with anti-myc or anti-PECAM as a negative control. Actin was detected (arrowhead) by anti-GFP antibodies in all the samples including cells transfected with truncated Kif1A. Inputs are shown in Supplementary Figure S7.

In order to test which domain contributes to the altered myoblast cell morphology, we constructed a series of truncated Kif1A proteins (Figure 5F) and overexpressed them in C2C12 myoblast cells by transfection with a mouse Kif1A expression construct. Control cells, transfected with GFP, exhibited rectangular, extended shapes with many mostly longitudinally oriented actin filaments (Figure 5A-A"). Cells overexpressing full-length Kif1A or Kif1A lacking the C terminal PH domain $(\triangle \mathrm{PH}$, see Figure $5 \mathrm{~F}$ for domain structure and truncated constructs) assumed broader, more expanded shapes (Figure 5B-B",C-C"). The F-actin staining shows a rearrangement of stress fibers to the periphery in Kif1A overexpressing cells (Figure 5B, open arrowhead). In addition, Kif1A protein (detected by the myc tag) in these overexpressing cells appeared to be unevenly distributed, forming isolated aggregations and localized densities (Figure $5 \mathrm{~B}^{\prime}, \mathrm{C}^{\prime}$, solid arrowheads). Interestingly, the large regions of F-actin displacement appear to be coincident with the larger Kif1A aggregations (Figure 5B"), and peripheral F-actin accumulations (Figure $5 \mathrm{~B}^{\prime}, \mathrm{C}^{\prime}$, open arrowheads) are coincident with the localized densities (Figure 5B', $\mathrm{C}^{\prime}$ solid arrowhead). In contrast, cells transfected with Kif1A lacking the MD or FHA domains showed distributions of actin filaments that were similar to control cells (Figure 5D-D",E-E") and the myc-tagged Kif1A protein was evenly distributed throughout the cells.

Expression of the MD, FHA, or PH domains by themselves does not produce any changes in cell morphology or actin fiber formation (Supplementary Figure S5). Interestingly, expression of MD alone results in the nuclear localization of this truncated protein (Supplementary Figure S6A'). It is likely that the FHA domain is responsible for the cytoplasmic localization of Kif1A since the $\triangle \mathrm{PH}$ construct (MD-FHA combination) is not found in the nucleus (Figure 5C").

To quantify an effect of Kif1A OE on cell morphology, we measured two-dimensional cell size normalized to the size of the nucleus. Kif1A overexpression resulted in a dramatic increased in size compared to GFP-transfected control cells $36 \mathrm{~h}$ after transfection (Figure 5G), suggesting that overexpression of Kif1A alters normal cell morphology. Overexpression of Kif1A that lacks the $\mathrm{PH}$ domain $(\triangle \mathrm{PH})$ resulted in a slight increase in cell size compared to GFP-expressing controls (Figure $\left.5 C-C^{\prime \prime}, G\right)$ but this increase was smaller than that observed following overexpression of the full-length protein. Overexpression of Kif1A lacking either the FHA domain ( $\mathrm{FHA}$ ) had little effect on cell size (Figure 5G), which was consistent with the lack of effect on morphology (Figure 5E-E"). Together, these data suggest that all three functional domains are required for the significantly increased cell size seen in response to overexpression of the full-length Kif1A.

We also tested the ability of Kif1A to directly bind actin. HEK293T cells were co-transfected with full-length or truncated myc-tagged Kif1 $A$ and GFP-tagged actin. Immunoprecipitation with anti-GFP antibodies pulled down full-length and all truncated forms of the myc-tagged Kif1A (Figure 5H and Supplementary Figure S7). Note that $\triangle \mathrm{PH}$ associates with G-actin more strongly than the other truncated Kif1A constructs, consistent with the stronger accumulation of $\triangle \mathrm{PH}$ at the edge of C2C12 
cells (see Figure 6C). Taken together, these results suggest that Kif1A affects actin filament organization and mammalian cell morphology.
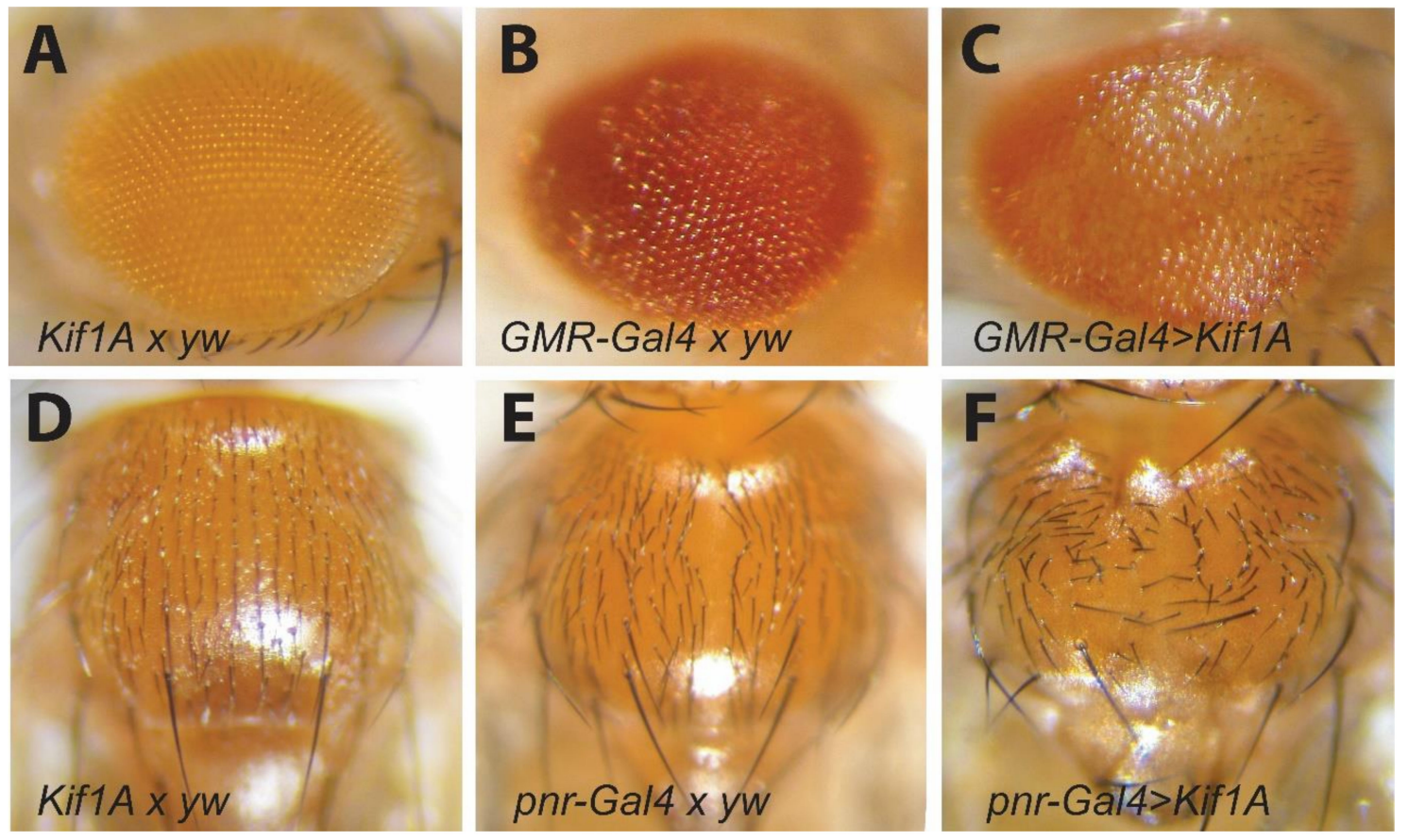

Figure 6. Kif1 $A$ overexpression impairs viability and cell polarity: Kif1A overexpression impairs viability of ommatidia and pigment cells. (A) One-week-old Kif1A x yw and (B) GMR-Gal4xyw controls. (C) Driven Kif1A OE in age-matched flies exhibited rougher and more decolorized external eyes compared to genetic controls. Kif1 $A$ overexpression impairs bristle arrangement. (D) One-week-old Kif1A x yw and (E) pnr x yw controls. (F) Overexpression of Kif1A in dorsal mesoderm using the Pnr-Gal4 driver resulted in disorientated bristle arrangement compared to genetic controls.

\subsection{Kif1A OE Affects Cell Viability and Cell Polarity}

We then determined whether the reorganization of the cellular actin cytoskeleton in both fly hearts and in cultured cardiomyocytes might also affect cell polarity. We took advantage of two model systems in the fly that provide readouts for alterations in cell polarity: ommatidium development in the fly eye [30] and bristle pattern formation in the thorax [31]. We overexpressed Kif1A specifically in photoreceptors and pigment cells in developing eyes using the GMR-Gal4 driver [32]. Eyes in Kif1A/yw outcrossed controls show the normal ommatidium arrangement with the expected white eye phenotype (Figure 6A). GMR-Gal4/yw controls show the expected red eye phenotype but are slightly glassy in appearance, likely the result of some developmental effects due to overexpression of Gal4 itself from this strong driver line (Figure 6B). In contrast, eyes from Kif1 Aa OE flies exhibited an edematous, glassy-eye appearance and disorganized cell arrangement (Figure 6C compared to Figure $6 \mathrm{~A}, \mathrm{~B})$. These phenotypes, along with the reduced red eye color, suggest a reduction in overall cell viability. We also overexpressed Kif1A in the dorsal part of the embryo using the Pannier (Pnr)-Gal4 driver [33]. Again, we observed the normal, regular arrangement of bristles in KIF1A/yw controls (Figure 6D) and a slight disruption of bristle orientation in the Pnr-Gal4/yw controls that may reflect Gal4 leakiness from this driver [34] (Figure 6E). However, bristle orientation in the Pnr > Kif1a flies was severely disrupted, indicating that Kif1 $A a$ overexpression also compromises cell polarity (Figure 5F).

\subsection{Overexpression of Kif1A Inhibits Muscle Maturation}

Embryonic analysis of cardiac precursor cells in mesodermal Kif1 $A$ overexpressing flies indicates that Kif1 $A$ does not affect the cardiac cell commitment (Supplementary Figure S3). However, because 
Kif1A altered actin organization in myoblast cells (Figure 5), we hypothesized that Kif1A may affect or redirect actin remodeling that occurs during myocyte maturation. To test this hypothesis, we initiated differentiation of the Kif1A-transfected C2C12 cells into myocytes in vitro (Figure 7). Cells transfected with the GFP control vector formed syncytia with abundant muscle myofibrils (Figure 7B). Kif1A-transfected cells also formed syncytia; however, they had very scant cytoplasm (Figure 7A"'). Quantification of the area of transfected cells reveals a significantly smaller total area per field of view and a smaller number of associated nuclei for cells transfected with Kif1 $A$ compared to GFP-transfected controls (Supplementary Figure S8). Interestingly, the ratio of cell area per nucleus was the same for both GFP and Kif1A-transfected cells. Taken together, these results suggest that overexpression of Kif1A impairs myocyte maturation, likely through disruption of actin organization, which is consistent with the effects of Kif1 $A$ overexpression in the fly heart.
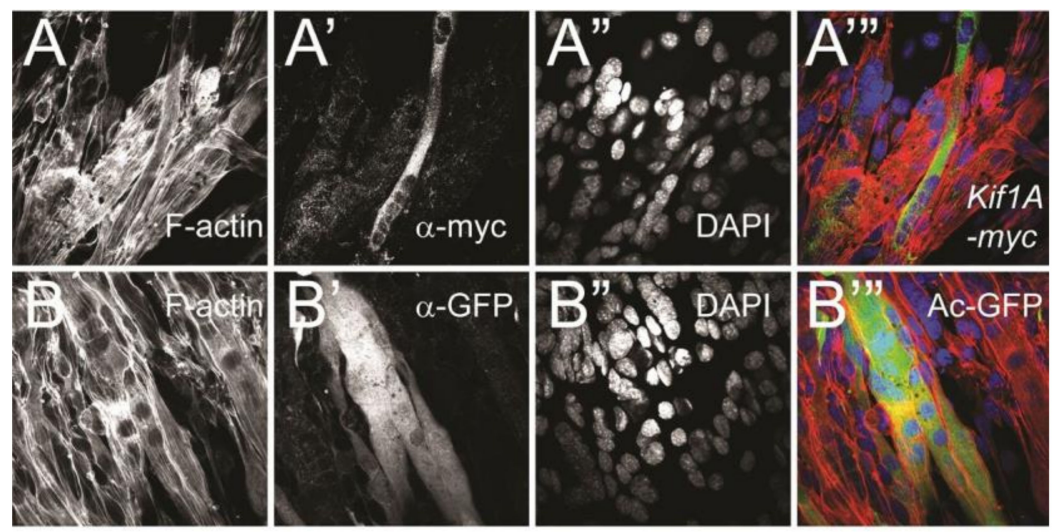

Figure 7. Kif1A overexpression affects muscle maturation: Twenty-four hours after transfection (with Kif1A-myc or AcGFP), C2C12 myoblast cells were switched to differentiation medium for $36 \mathrm{~h}$ and then fixed and stained. (A-A"') Kif1A transfected cells. (B-B") GFP-transfected cells. (A,B) Phalloidin staining reveals F-actin organization in all cells. ( $\left.\mathbf{A}^{\prime}\right)$ Anti-myc staining to detect Kif1A expressing cells and (B') anti-GFP staining to detect GFP-transfected control cells. ( $\left.\mathbf{A}^{\prime \prime}, \mathbf{B}^{\prime \prime}\right)$ DAPI nuclear staining. $\left(\mathbf{A}^{\prime \prime \prime}, \mathbf{B}^{\prime \prime \prime}\right)$ Merged images: note that differentiated C2C12 cells with Kif1A overexpression (A,C-C'") formed a syncytium but contained scant cytoplasm.

Previous studies have demonstrated that the Kif1A protein directly interacts with tubulin in microtubules $[23,35]$. Therefore, we examined the distribution and morphology of actin and microtubules (tubulin) in $\mathrm{C} 2 \mathrm{C} 12$ cells where Kif1 $A$ was overexpressed. We found no apparent morphological changes in size and distribution of actin (Supplementary Figure S5) or tubulin (Supplementary Figure S6) when Kif1A is overexpressed. This suggests that excess levels of Kif1A impair cardiac development primarily due to its effect on actin organization

\section{Discussion}

In this study, we identify the Kif1 $A$ gene as a candidate gene for causing left-sided heart defects and utilize Drosophila to demonstrate the pathologic effects of overexpression of the Kif1A gene on heart development and function. We demonstrate that, while KD of Kif1A had no effect on heart development or function, ectopic expression of Kif1 $A$ in the Drosophila heart causes dysplastic valves and cardiac remodeling associated with diminished contractile capability (Figures 2 and 3). Interestingly, these observations are among the hallmark features of one of the most severe human congenital heart defects, hypoplastic left heart syndrome (HLHS). Although the balanced translocation in this patient with left-sided heart defects may be a coincidental finding, the observation in this study that overexpression of Kif1 $A$ in Drosophila phenocopies at least one of the primary cardiac defects in the patient, i.e., aortic valve disease, is compelling. 
Although disease-associated mutations often cause loss of function, mutations causing overexpression of the regulator of calcineurin have previously been linked to sporadic congenital heart disease [30] and overexpression of limb-bud and heart, a regulator of transcription, in the mouse heart recapitulates $\mathrm{CHD}$ reported in humans with partial trisomy $2 p$ syndrome [36]. Consistent with previous findings in neural cells [16,37], our studies also indicate that exogenously expressed Kif1A protein accumulates at the edge of muscle cells. Our cell culture experiments suggest that this effect requires the MD and FHA domains of the Kif1A protein. The requirement for MD (Figure 5) suggests that the effect is exerted as the Kif1A protein translocates along microtubules. Combined expression of the MD together with FHA domain $(\triangle \mathrm{PH})$ caused accumulation of protein at the leading edge of the cell, suggesting that it is the $\mathrm{pH}$ domain that normally prevents this Kif1 A accumulation (Figure $5 \mathrm{C}^{\prime}$ ). A possible link between Kif1A and actin organization may lie in Kif1A's ability to interact directly with actin (Supplementary Figure S8). Kif1A mislocalization/aggregation may sequester G-actin and interfere with F-actin formation and organization. Previous studies have demonstrated that actin mutations causing malformation of the cytoskeleton lead to the development of cardiomyopathies [38,39]. Recently, mutations in the alpha cardiac actin gene were identified in association with familial atrial septal defects [38], suggesting an important role for cardiac actin in cardiac development. Our studies demonstrate that overexpression of Kif1 $A$ during cardiac development results in structural heart defects, likely by affecting actin organization.

\section{Pathological Effects Associated with Kif1A Overexpression in the Heart}

Based on the identification of a balanced translocation near Kif1 $A$ in a patient with left-sided heart defects, we used the Drosophila heart model to manipulate Kif1A protein levels. We found that overexpression of Kif1A in cardiomyocytes in the fly had a striking effect on the myofibrillar structure, cardiac contractility, fibrosis, and valve differentiation. These data are strikingly consistent with the histopathology in the patient's valve abnormalities and fibrosis (Figure 1), as well as what has been described in a subset of human HLHS hearts [5,40,41].

In summary, we have identified a patient with left-sided heart defects in association with a $\mathrm{t}(2 ; 8)(\mathrm{q} 37 ; \mathrm{p} 11)$ balanced translocation. Molecular mapping of the $2 \mathrm{q} 37$ translocation breakpoint, a locus previously associated with left-sided obstructive lesions, implicated the KIF1A gene as a candidate gene for causing these defects. In this study, we demonstrated that overexpression of Kif $1 A$ in the Drosophila heart caused morphological changes reminiscent of those that occur in HLHS. Specifically, our findings suggest that disruption of the F-actin organization causes ventricular hypoplasia with decreased function due to a primary defect in ventricular development in addition to an accompanying defect in valve development. Future studies in mammalian animal model systems should yield important new insights into the role of the F-actin organization in causing left-sided heart defects.

Supplementary Materials: The following are available online at http://www.mdpi.com/2308-3425/7/2/22/s1, Supplementary Table S1. Quantification of function in hearts from control and Kif1A overexpression and knockdown, Supplementary Figure S1. Unc expression, Supplementary Figure S2. Kif1A overexpression causes systolic dysfunction, Supplementary Figure S3. Western Blot analysis, Supplementary Figure S4. Cardiac primordial cell arrangement is not affected during embryogenesis, Supplementary Figure S5. Actin cytoskeleton is not affected by overexpression of the individual Kif1A functional domains, Supplementary Figure S6. Microtubule structure is unaffected by Kif1A overexpression, Supplementary Figure S7. Kif1A physically associates with G-actin in myocytes, Supplementary Figure S8. Quantification of Kif1A-transfected C2C12 induced myocytes, Video S1. High-speed movies of in situ Drosophila hearts.

Author Contributions: Conceptualization, T.A., R.B., P.G., and K.O.; methodology, T.A., L.L., K.O., and G.V.; investigation, T.A., L.L., K.O., and G.V.; formal analysis, T.A., L.L., K.O., and G.V.; writing一original draft preparation, T.A., R.B., P.G., and K.O.; writing—review and editing, T.A., R.B., P.G., and K.O.; project administration, T.A., R.B., P.G., and K.O.; supervision, T.A., R.B., P.G., and K.O.; funding acquisition, R.B. and K.O. All authors have read and agreed to the published version of the manuscript.

Funding: This research was funded by grants from the American Heart Association (14GRNT20490239) to K.O., from the National Institutes of Health (HL54732) to R.B., and from the National Institutes of Health (HL132241) to K.O. 
Acknowledgments: The authors would like to thank Grant Hogg for his technical assistance; M. Benjamin Perryman for contributions to early stages of this research; Andrew Nicholson, Department of Histopathology, the Royal Brompton Hospital, London, England, for providing the paraffin-embedded human heart valve tissue from the patient; and Jonathan Lucas, San Diego County Coroner's Office, for providing human aortic valve control tissue.

Conflicts of Interest: The funders had no role in the design of the study; in the collection, analyses, or interpretation of data; in the writing of the manuscript; or in the decision to publish the results.

\section{References}

1. Abdulla, R. Perspective in pediatric cardiology. Volume 5. Genetic and environmental risk factors of major cardiovascular malformations. Pediatr. Cardiol. 1998, 19. [CrossRef] [PubMed]

2. Grossfeld, P.D. The genetics of congenital heart disease. J. Nucl. Cardiol. 2003, 10, 71-76. [CrossRef] [PubMed]

3. Grossfeld, P.D. The genetics of hypoplastic left heart syndrome. Cardiol. Young 1999, 9, 627-632. [CrossRef] [PubMed]

4. deAlmeida, A.; McQuinn, T.; Sedmera, D. Increased ventricular preload is compensated by myocyte proliferation in normal and hypoplastic fetal chick left ventricle. Circ. Res. 2007, 100, 1363-1370. [CrossRef] [PubMed]

5. Bohlmeyer, T.J.; Helmke, S.; Ge, S.; Lynch, J.; Brodsky, G.; Sederberg, J.H.; Robertson, A.D.; Minobe, W.; Bristow, M.R.; Perryman, M.B. Hypoplastic left heart syndrome myocytes are differentiated but possess a unique phenotype. Cardiovasc. Pathol. 2003, 12, 23-31. [CrossRef]

6. Elliott, D.A.; Kirk, E.P.; Yeoh, T.; Chandar, S.; McKenzie, F.; Tayor, P.; Grossfeld, P.; Fatkin, D.; Jones, O.; Hayes, P.; et al. Cardiac homeobox gene NKX2-5 mutations and congenital heart disease: Associations with atrial septal defect and hypoplastic left heart syndrome. J. Am. Coll. Cardiol. 2003, 41, 2072-2076. [CrossRef]

7. Pashmforoush, M.; Lu, J.T.; Chen, H.; Amad, T.S.; Kondo, R.; Pradervand, S.; Evans, S.M.; Clark, B.; Feramisco, J.R.; Giles, W.; et al. Nkx2-5 pathways and congenital heart disease; loss of ventricular myocyte lineage specification leads to progressive cardiomyopathy and complete heart block. Cell 2004, 117, 373-386. [CrossRef]

8. Hirokawa, N. The mechanisms of fast and slow transport in neurons: Identification and characterization of the new kinesin superfamily motors. Curr. Opin. Neurobiol. 1997, 7, 605-614. [CrossRef]

9. Hirokawa, N.; Nitta, R.; Okada, Y. The mechanisms of kinesin motor motility: Lessons from the monomeric motor KIF1A. Nature reviews. Mol. Cell Boil. 2009, 10, 877-884.

10. Miki, H.; Okada, Y.; Hirokawa, N. Analysis of the kinesin superfamily: Insights into structure and function. Trends Cell Biol. 2005, 15, 467-476. [CrossRef]

11. Marszalek, J.R.; Ruiz-Lozano, P.; Roberts, E.; Chien, K.R.; Goldstein, L.S. Situs inversus and embryonic ciliary morphogenesis defects in mouse mutants lacking the KIF3A subunit of kinesin-II. Proc. Natl. Acad. Sci. USA 1999, 96, 5043-5048. [CrossRef] [PubMed]

12. Wang, Y.; Pan, X.; Fan, Y.; Hu, X.; Liu, X.; Xiang, M.; Wang, J. Dysregulated expression of microRNAs and mRNAs in myocardial infarction. Am. J. Transl Res. 2015, 7, 2291-2304. [PubMed]

13. Yoshikawa, K.; Kuwahara, M.; Saigoh, K.; Ishiura, H.; Yamagishi, Y.; Hamano, Y.; Samukawa, M.; Suzuki, H.; Hirano, M.; Mitsui, Y.; et al. The novel de novo mutation of KIF1A gene as the cause for Spastic paraplegia 30 in a Japanese case. eNeurologicalSci 2019, 14, 34-37. [CrossRef] [PubMed]

14. Ocorr, K.; Reeves, N.L.; Wessells, R.J.; Fink, M.; Chen, H.S.; Akasaka, Y.; Yasuda, S.; Metzger, J.M.; Giles, W.; Posakony, J.W.; et al. KCNQ potassium channel mutations cause cardiac arrhythmias in Drosophila that mimic the effects of aging. Proc. Natl. Acad. Sci. USA 2007, 104, 3943-3948. [CrossRef] [PubMed]

15. Ocorr, K.; Fink, M.; Cammarato, A.; Bernstein, S.; Bodmer, R. Semi-automated Optical Heartbeat Analysis of Small Hearts. JoVE 2009, 35, 1435. [CrossRef] [PubMed]

16. Fink, M.; Callol-Massot, C.; Chu, A.; Ruiz-Lozano, P.; Izpisua-Belmonte, J.C.; Giles, W.; Bodmer, R.; Ocorr, K. A new method for detection and quantification of heartbeat parameters in Drosophila, zebrafish, and embryonic mouse hearts. BioTechniques 2009, 46, 101-113. [CrossRef]

17. Alayari, N.N.; Vogler, G.; Taghli-Lamallem, O.; Ocorr, K.; Bodmer, R.; Cammarato, A. Fluorescent labeling of Drosophila heart structures. JoVE 2009, 32. [CrossRef] 
18. Doherty, E.S.; Lacbawan, F.L. $2 q 37$ Microdeletion Syndrome; Adam, M.P., Ardinger, H.H., Pagon, R.A., Wallace, S.E., Bean, L.J.H., Stephens, K., Amemiya, A., Eds.; GeneReviews: Seattle University of Washington: Seattle, WA, USA, 1993; pp. 1993-2020. Available online: https:/www.ncbi.nlm.nih.gov/books/NBK1158/ (accessed on 30 May 2020).

19. Neely, A.; Wei, X.; Olcese, R.; Birnbaumer, L.; Stefani, E. Potentiation by the beta subunit of the ratio of the ionic current to the charge movement in the cardiac calcium channel. Science 1993, 262, 575-578. [CrossRef]

20. Taghli-Lamallem, O.; Akasaka, T.; Hogg, G.; Nudel, U.; Yafee, D.; Chamberlain, J.S.; Ocorr, K.; Bodmer, R. Dystrophin deficiency in Drosophila reduces lifespan and causes a dilated cardiomyopathy phenotype. Aging Cell 2008, 7, 237-249. [CrossRef]

21. Cammarato, A.; Dambacher, C.M.; Knowles, A.F.; Kronert, W.A.; Bodmer, R.; Ocorr, K.; Bernstein, S.I. Myosin transducer mutations differentially affect motor function, myofibril structure, and the performance of skeletal and cardiac muscles. Mol. Biol. Cell 2008, 19, 553-562. [CrossRef]

22. Akasaka, T.; Klinedinst, S.; Ocorr, K.; Busamante, E.; Kim, S.K.; Bodmer, R. The ATP-sensitive potassium (KATP) channel-encoded dSUR gene is required for Drosophila heart function and is regulated by tinman. Proc. Natl. Acad. Sci. USA 2006, 103, 11999-12004. [CrossRef] [PubMed]

23. Okada, Y.; Yamazaki, H.; Sekine-Aizawa, Y.; Hirokawa, N. The neuron-specific kinesin superfamily protein KIF1A is a unique monomeric motor for anterograde axonal transport of synaptic vesicle precursors. Cell 1995, 81, 769-780. [CrossRef]

24. Brand, A.H.; Perrimon, N. Targeted gene expression as a means of altering cell fates and generating dominant phenotypes. Development 1993, 118, 401-415. [PubMed]

25. Yonekawa, Y.; Harada, A.; Okada, Y.; Funakoshi, T.; Kanai, Y.; Takei, Y.; Noda, T.; Hirokawa, N. Defect in synaptic vesicle precursor transport and neuronal cell death in KIF1A motor protein-deficient mice. J. Cell Biol. 1998, 141, 431-441.

26. Curtis, N.J.; Ringo, J.M.; Dowse, H.B. Morphology of the pupal heart, adult heart, and associated tissues in the fruit fly, Drosophila melanogaster. J. Morphol. 1999, 240, 225-235. [CrossRef]

27. Molina, M.R.; Cripps, R.M. Ostia, the inflow tracts of the Drosophila heart, develop from a genetically distinct subset of cardial cells. Mech. Dev. 2001, 109, 51-59. [CrossRef]

28. Lee, J.R.; Shin, H.; Choi, J.; Ko, J.; Kim, S.; Lee, H.W.; Kim, K.; Rho, S.H.; Lee, J.H.; Song, H.E.; et al. An intramolecular interaction between the FHA domain and a coiled coil negatively regulates the kinesin motor KIF1A. EMBO 2004, 23, 1506-1515. [CrossRef]

29. Klopfenstein, D.R.; Tomishige, M.; Stuurman, N.; Vale, R.D. Role of phosphatidylinositol $(4,5)$ bisphosphate organization in membrane transport by the Unc104 kinesin motor. Cell 2002, 109, 347-358. [CrossRef]

30. Liu, M.A.; Huang, A.M.; Chou, C.K.; Liaw, G.J.; Wu, C.W. Utilization of Drosophila eye to probe the functions of two mammalian serine/threonine kinases, Snk and HsHPK. J. Biomed. Sci. 2001, 8, 270-277. [CrossRef]

31. Calleja, M.; Renaud, O.; Usui, K.; Pistillo, D.; Morata, G.; Simpson, P. How to pattern an epithelium: Lessons from achaete-scute regulation on the notum of Drosophila. Gene 2002, 292, 1-12. [CrossRef]

32. Moses, K.; Rubin, G.M. Glass encodes a site-specific DNA-binding protein that is regulated in response to positional signals in the developing Drosophila eye. Genes Dev. 1991, 5, 583-593. [CrossRef] [PubMed]

33. Heitzler, P.; Haenlin, M.; Ramain, P.; Calleja, M.; Simpson, P. A genetic analysis of pannier, a gene necessary for viability of dorsal tissues and bristle positioning in Drosophila. Genetics 1996, 143, 1271-1286. [PubMed]

34. Yoshioka, Y.; Nguyen, T.T.; Fujiwara, S.; Matsuda, R.; Valdez-Graham, V.; Zurita, M.; Yamaguchi, M. Drosophila DREF acting via the JNK pathway is required for thorax development. Genesis 2012, 50, 599-611. [CrossRef] [PubMed]

35. Aprodu, I.; Soncini, M.; Redaelli, A. Interaction forces and interface properties of KIF1A kinesin-alphabeta tubulin complex assessed by molecular dynamics. J. Biomech. 2008, 41, 3196-3201. [CrossRef]

36. Li, X.; Shi, L.; Xu, M.; Zheng, X.; Yu, Y.; Jin, J. RCAN1 Mutation and Functional Characterization in Children with Sporadic Congenital Heart Disease. Pediatr. Cardiol. 2018, 39, 226-235. [CrossRef]

37. Briegel, K.J.; Baldwin, H.S.; Epstein, J.A.; Joyner, A.L. Congenital heart disease reminiscent of partial trisomy 2p syndrome in mice transgenic for the transcription factor Lbh. Development 2005, 132, 3305-3316. [CrossRef]

38. Harvey, P.A.; Leinwand, L.A. The cell biology of disease: Cellular mechanisms of cardiomyopathy. J. Cell Biol. 2011, 194, 355-365. [CrossRef] 
39. Debold, E.P.; Saber, W.; Cheema, Y.; Bookwalter, C.S.; Trybus, K.M.; Warshaw, D.M.; Vaburen, P. Human actin mutations associated with hypertrophic and dilated cardiomyopathies demonstrate distinct thin filament regulatory properties in vitro. J. Mol. Cell Cardiol. 2010, 48, 286-292. [CrossRef]

40. Gaber, N.; Gagliardi, M.; Patel, P.; Kinnear, C.; Zhang, C.; Chitaya, D.; Shannon, P.; Jaeggi, E.; Tabouri, U.; Keller, G.; et al. Fetal reprogramming and senescence in hypoplastic left heart syndrome and in human pluripotent stem cells during cardiac differentiation. Am. J. Pathol. 2013, 183, 720-734. [CrossRef]

41. Cole, C.R.; Eghtesady, P. The myocardial and coronary histopathology and pathogenesis of hypoplastic left heart syndrome. Cardiol. Young 2016, 26, 19-29. [CrossRef]

(C) 2020 by the authors. Licensee MDPI, Basel, Switzerland. This article is an open access article distributed under the terms and conditions of the Creative Commons Attribution (CC BY) license (http://creativecommons.org/licenses/by/4.0/). 